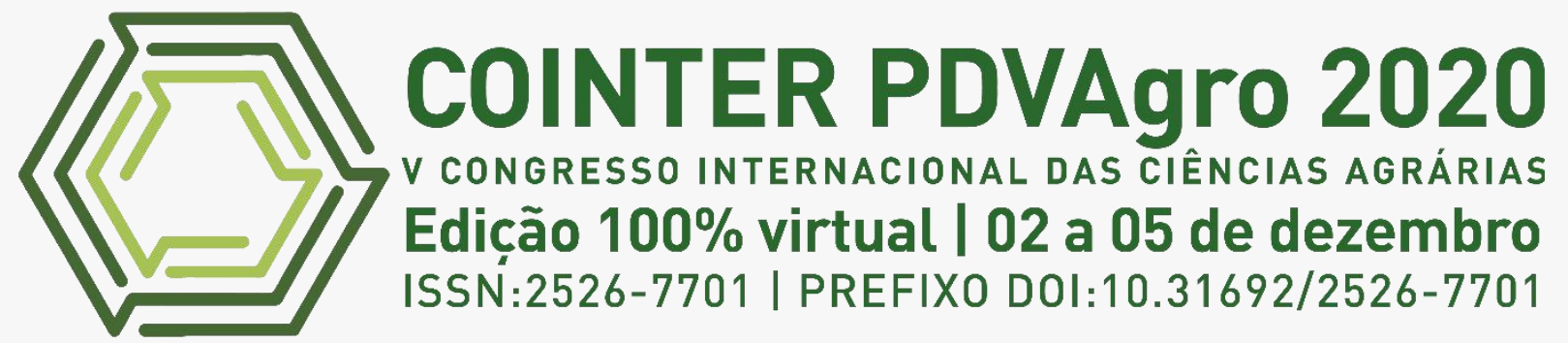

\title{
VIABILIDADE DO ARMAZENAMENTO DE SEMENTES DE Oenocarpus mapora H. KARSTEN EM DIFERENTES RECIPIENTES
}

\section{VIABILIDAD DEL ALMACENAMIENTO DE SEMILLAS DE Oenocarpus mapora H. KARSTEN EN DIFERENTES CONTENEDORES}

\section{VIABILITY OF Oenocarpus mapora H. KARSTEN SEED STORAGE IN DIFFERENT CONTAINERS}

\author{
Apresentação: Comunicação Oral
}

\begin{abstract}
Sinara de Nazaré Santana Brito ${ }^{1}$; Harleson Sidney Almeida Monteiro²; Antonia Benedita da Silva Bronze ${ }^{3}$, Igor
\end{abstract} Cristian de Oliveira Vieira ${ }^{4}$; André Santana Bezerra ${ }^{5}$

DOI: https://doi.org/10.31692/2526-7701.VCOINTERPDVAgro.0514

\begin{abstract}
RESUMO
A espécie Oenocarpus mapora H. Karsten, conhecida popularmente como bacabi é uma palmeira nativa da Amazônia, rústica e pouco explorada comercialmente, mas com grande potencial econômico para produção de frutos e palmito, utilizados em polpas, doces, sucos, sorvetes, cosméticos e pela propriedade oleaginosa, semelhantes ao azeite de oliva. As pesquisas com essa palmeira, ainda são incipientes, principalmente relacionadas a germinação e meios para armazenagem da semente, as quais são recalcitrantes. Haja vista que, para conservar a qualidade das sementes, é necessário o armazenamento. E o armazenamento para que seja eficiente, é imprescindível o conhecimento sobre a fisiologia da semente, teor de umidade, e as suas respectivas mudanças hormonais e consequentemente o vigor, sendo esses fatores indispensáveis para prolongar ou acelerar o processo de deterioração das sementes durante o armazenamento e assim conservar a qualidade das mesmas. Sendo assim, objetivou-se avaliar o comportamento de sementes de Oenocarpus mapora $\mathrm{H}$. Karsten em relação ao armazenamento em diferentes recipientes visando a conservação e germinação. Os frutos de Bacabi foram coletados no município de Santo Antônio do Tauá/Pará, em uma propriedade rural, colhidos a partir de uma matriz com cachos maduros. Foram testados 7 recipientes para armazenamento das sementes: T1 (vidro), T2 (saco plástico), T3 (saco de papel), T4 (garrafa PET), T5 (saco plástico com areia), T6 (saco plástico com serragem) e T7 (saco plástico preto). A avaliação foi feita em três períodos 15, 57, 99 dias após armazenamento, analisando as variáveis: Teor de Água na semente (TA\%) e Percentagem de germinação (G\%). O delineamento do experimento foi inteiramente casualizado em esquema fatorial de $7 \mathrm{x} 3$ (tratamentos $\mathrm{x}$ períodos). Os resultados obtidos mostraram que a viabilidade das sementes de bacabi é afetada pelo tipo e pelo período de armazenamento no recipiente e observou-se que os melhores tratamentos foram o T2 (saco plástico), T4 (garrafa PET), T5

\footnotetext{
${ }^{1}$ Graduanda em Agronomia, Universidade Federal Rural da Amazônia, sinaraagroufra@gmail.com

${ }^{2}$ Graduando em Agronomia, Universidade Federal Rural da Amazônia, harlesonsamonteiro@gmail.com

${ }^{3}$ Doutora, Universidade Federal Rural da Amazônia, antonia.silva@ufra.edu.br

${ }^{4}$ Mestrando em Agronomia (Ciência do Solo), Universidade Estadual Paulista "Júlio Mesquita Filho", igor.vieira@unesp.br

${ }^{5}$ Engenheiro Agrônomo, Universidade Federal Rural da Amazônia, andrebezerra.live@gmail.com
} 
(saco plástico com areia) e T7 (saco plástico preto), pois preservaram as qualidades fisiológicas da semente durante o armazenamento por mais tempo, sem alterar a germinação, pois preservam as qualidades fisiológicas da semente de bacabi durante o armazenamento por mais tempo, sem alterar a germinação posterior.

Palavras-Chave: Fruticultura, Bacabizeiro, Armazenamento, Amazônia

\title{
RESUMEN
}

La especie Oenocarpus mapora H. Karsten, conocida popularmente como bacabi es una palma originaria de la Amazonía, rústica y poco explotada comercialmente, pero con gran potencial económico para la producción de frutos y palmito, utilizada en pulpas, dulces, jugos, helados, cosméticos y propiedad oleaginosa, similar al aceite de oliva. La investigación con esta palma es aún incipiente, principalmente relacionada con la germinación y los medios de almacenamiento de la semilla, que son recalcitrantes. Teniendo en cuenta que, para preservar la calidad de las semillas, es necesario el almacenamiento. Y almacenamiento para que sea eficiente, es fundamental conocer la fisiología de la semilla, el contenido de humedad y sus respectivos cambios hormonales y en consecuencia el vigor, siendo estos factores indispensables para prolongar o acelerar el proceso de deterioro de las semillas durante el almacenamiento. y así preservar su calidad. Así, el objetivo fue evaluar el comportamiento de semillas de Oenocarpus mapora H. Karsten en relación al almacenamiento en diferentes recipientes para conservación y germinación. Los frutos de bacabi se recolectaron en el municipio de Santo Antônio do Tauá / Pará, en una propiedad rural, recolectados de una matriz con racimos maduros. Se probaron siete contenedores de almacenamiento de semillas: T1 (vidrio), T2 (bolsa de plástico), T3 (bolsa de papel), T4 (botella de PET), T5 (bolsa de plástico con arena), T6 (bolsa de plástico con aserrín) y T7 (bolsa de plástico negra). La evaluación se realizó en tres períodos 15, 57, 99 días después del almacenamiento, analizando las variables: Contenido de agua en la semilla $(\mathrm{TA} \%)$ y Porcentaje de germinación $(\mathrm{G} \%)$. El diseño del experimento fue completamente al azar en un esquema factorial de $7 \times 3$ (tratamientos x períodos). Los resultados obtenidos mostraron que la viabilidad de las semillas de bacabi se ve afectada por el tipo y período de almacenamiento en el contenedor y se observó que los mejores tratamientos fueron T2 (bolsa plástica), T4 (botella PET), T5 (bolsa plástica con arena ) y T7 (bolsa plástica negra), ya que preservan las cualidades fisiológicas de la semilla durante el almacenamiento por más tiempo, sin alterar la germinación, ya que preservan las cualidades fisiológicas de la semilla de bacabi durante el almacenamiento por más tiempo, sin alterar la germinación posterior.

Palabras clave: Fruta, Bacabizeiro, Almacenamiento, Amazon

\begin{abstract}
The species Oenocarpus mapora H. Karsten, popularly known as bacabi is a palm native to the Amazon, rustic and little commercially exploited, but with great economic potential for the production of fruits and hearts of palm, used in pulps, sweets, juices, ice cream, cosmetics and oleaginous property, similar to olive oil. Research with this palm is still incipient, mainly related to germination and means for storing the seed, which are recalcitrant. Bearing in mind that, to preserve the quality of the seeds, storage is necessary. And storage for it to be efficient, it is essential to know about the seed physiology, moisture content, and their respective hormonal changes and consequently the vigor, these factors being indispensable to prolong or accelerate the process of deterioration of the seeds during storage and thus preserve their quality. Thus, the objective was to evaluate the behavior of Oenocarpus mapora $\mathrm{H}$. Karsten seeds in relation to storage in different containers for conservation and germination. Bacabi fruits were collected in the municipality of Santo Antônio do Tauá / Pará, on a rural property, harvested from a matrix with mature clusters. Seven seed storage containers were tested: T1 (glass), T2 (plastic bag), T3 (paper bag), T4 (PET bottle), T5 (plastic bag with sand), T6 (plastic bag with sawdust) and T7 (black plastic bag). The evaluation was done in three periods 15, 57, 99 days after storage, analyzing the variables: Water content in the seed (TA\%) and Germination percentage $(\mathrm{G} \%)$. The design of the experiment was completely randomized in a $7 \times 3$ factorial scheme (treatments $x$ periods). The results obtained showed that the viability of bacabi seeds is affected by the type and period of storage in the container and it was observed that the best treatments were T2 (plastic bag),
\end{abstract}


T4 (PET bottle), T5 (plastic bag with sand ) and T7 (black plastic bag), as they preserved the physiological qualities of the seed during storage for a longer time, without altering germination, as they preserve the physiological qualities of the bacabi seed during storage for a longer time, without altering later germination.

Keywords: Fruit, Bacabizeiro, Storage, Amazon

\section{INTRODUÇÃO}

A espécie Oenocarpus mapora H. Karsten, conhecida popularmente como bacabi, é uma palmeira perene nativa da Amazônia, encontrada ainda em alguns países da América Central, e esta espécie é rústica e pouco explorada, apresentando um hábito de crescimento em forma de touceira (HENDERSON et.al 1995).

Essa espécie pertence à Família Arecaceae, terceira mais importante para os habitantes da região Amazônica por possuir um grande número de espécies de interesse que compõe a alimentação dos mesmos, acrescentando mais fonte de alimentação, principalmente aos povos ribeirinhos (DOMINGUES, 2014). O gênero Oenocarpus possui características peculiares, seja no formato, que vai de elíptico a globoso, apresentando coloração roxa-escura quando estão maduros, além de ter um grande potencial para produção de frutos e palmito (SILVA, 2006). Uma vez que, a palmeira apresenta caracteristicas semelhantes ao açaizeiro (Euterpe oleracea), que na ausência de chacos e frutos, pode ser confundida, assim como, suas semelhanças estaão em altura, diametro, folha e folíolos (QUEIROZ \& BIANCO, 2009).

No Brasil a espécie é comumente encontrada no alto Amazonas, no Amapá e no Pará, em locais de mata e capoeira, com utilização para o cultivo agrícola e, em amplas áreas de cultivo. Os frutos têm usos similares aos do açaizeiro, e permitem a extração de óleo semelhante ao óleo de oliva, possuem consumo mais restrito, somente pelas populações locais, contudo as espécies que perfilham podem ser promissoras para a produção de palmito e para o artesanato (OLIVEIRA \& RIOS, 2014).

As pesquisas ainda são incipientes, estudos e trabalhos com germinação e meios para armazenagem da semente de bacabi, que tem comportamento recalcitrante, ou seja, apresentam perda drástica ou total da capacidade de germinação, quando o grau de umidade é reduzido para nível igual ou superior a 15\% (CARVALHO; MULLER; NASCIMENTO, 2001).

A embalagem é um fator fundamental para a obtenção de sucesso no armazenamento das sementes, pois são importantes não só para separar os diferentes lotes, como também para proteger as sementes contra insetos e animais, e ainda facilitar o manejo e aproveitar melhor o espaço de armazenamento (CROCHEMORE, 1993). A decisão a ser tomada quanto ao tipo de embalagem que deverá ser utilizada, depende da natureza da semente, do método de 
armazenamento e do tempo em que a semente ficará armazenada. Portanto, quanto mais favoráveis forem as condições de armazenamento e umidade, maior será o período de conservação (SILVA et al., 2009).

Para conservar a qualidade das sementes, é necessário o armazenamento. E o armazenamento para que seja eficiente, é imprescindível o conhecimento sobre a fisiologia da semente, teor de umidade, e as suas respectivas mudanças hormonais e consequentemente o vigor, sendo esses fatores indispensáveis para prolongar ou acelerar o processo de deterioração das sementes durante o armazenamento e assim conservar a qualidade das mesmas (BORÉM, 2007).

A boa e adequada conservação das sementes, reflete na qualidade e viabilidade das sementes, que vão gerar mudas saudáveis que se transformarão em plantas adultas de qualidade, sadias e produtivas (SILVA et al., 2010). Portanto o armazenamento adequado é de grande importância, para transporte de sementes até as localidades distantes, pois nesses casos deseja-se que a semente não germine durante esse período (MEDEIROS, 2001).

O trabalho teve como objetivo avaliar o comportamento de sementes de Oenocarpus mapora H. Karsten em relação ao armazenamento em diferentes recipientes visando a conservação e germinação.

\section{FUNDAMENTAÇÃO TEÓRICA}

A Oenocarpus mapora H. Karsten é uma palmeira perene pertecente da biodiversidade, originária da América Tropical, sendo de maior ocorrência no Norte da América do sul, que expressa precocidade na produção de cachos, com frutos de excelente composição química e nutricional, contribuindo no enriquecimento de vitaminas na alimentação dos povos tradicionais (OLIVEIRA; MOURA, 2010).

A Família Arecaceae possui uma distribuição bastante diferenciada, sendo constituída de 1500 espécies distribuídas em 200 gêneros pertencentes a seis subfamílias que se encontram por toda a região tropical do planeta. Nesta família acha-se plantas com portes arbustivos, arbóreos e as trepadeiras quase que raramente (SILVA, 2006).

A amazônia possui uma diversidade de espécies com elevado potencial econômico, nutricional e social que, necessitam ser domesticadas e genéticamente melhoradas, dentre elas está o Oenocarpus mapora Mart., mas conhecida como bacabi (MIRANDA et al., 2001).

Os frutos são comestíveis e muito apreciados pelas comunidades indígenas e caboclas da Amazônia, sendo a partir deles preparada polpa de bacabi, de sabor agradável semelhante 
ao do açaizeiro e com alto teor de óleo que é extraído do endocarpo dos frutos (OLIVEIRA et al, 2014).

Atualmente existe uma demanda mundial por alternativas de óleos e gorduras saudáveis, e para isso seja viável é indispensavel utilizar matéria prima e tecnologias de processamento para geração de conhecimento, e obter uma produção de acordo com a demanda de mercado, e o bacabi é uma espécie que apresenta possibilidade de uso integral do fruto, que por sua vez tem uma excelente composição química e é rico em proteínas (BRONZE et al., 2011).

Apesar do O. Mapora possuir boas perspectivas comerciais, apresenta alguns entraves em seu cultivo em larga escala e na sua domesticação que estão atrelados ao manejo e melhoramento genético inerentes a biologia floral, biologia reprodutiva e na ecologia da reprodução (OLIVEIRA; PADILHA; FERNANDES, 2002).

Tendo em vista, as características peculiares da espécie supracitadas, vale ressaltar que as sementes possuem comportamento recalcitrantes, não toleram perda de água, caso ocorra tal fato as sementes perdem sua viabilidade em poucas horas, e o teor de água em torno de 26,6\% impossibilitam a sua geminação, sendo este um fator de extrema relevância para cultivos racionais (JOSÉ; ERASMO; COUTINHO, 2012).

O tipo recalcitrantes são um grupo de sementes que se diferem das ortodoxas, não suportando o armazenamento a temperaturas negativas, chegando a perder a viabilidade. Dessa forma, a longevidade e viabilidade dessas sementes mesmo em condições bastante favoráveis, ainda é bastante curta (ROBERTS, 1972).

O dessecamento é um fator limitante para germinação de sementes de palmeiras, devido a sua sensibilidade a este processo, o que ocasiona a perda de sua viabilidade germinativa, o que acarreta dificuldades na emergência de sementes de palmeiras (SILVA; MOTA; FARIAS NETO, 2009).

As sementes das palmeiras apresentam sinais de dormência, e crescimento embrionário sucessivo e lento, e estas peculiaridades podem está correlacionadas à sua capacidade de armazenar as reservas orgânicas e, em seguida mobilizar para o seu crescimento (CUNHA \& JARDIM, 1995).

Diversos estudos mostram que a conservação que ocorre das sementes durante o processo de colheita e a semeadura, interferem na qualidade e na quantidade das plântulas que serão obtidas e, bem como, no desempenho produtivo da população estabelecida no campo. O conhecimento disponível, particularmente a respeito da conservação de sementes de espécies nativas em fase de domesticação não é suficiente para embasar a definição de 
tecnologias de

Armazenamento (NASCIMENTO, 2006).

De um modo geral, o armazenamento de sementes recalcitrantes deve proporcionar condições que previnam a perda de água, permitam o controle de microrganismos patogênicos e mantenham o suprimento adequado de oxigênio (MENDONÇA et al., 2012). Para Bonner (1990), a embalagem empregada deve ser impermeável ao vapor de água e, ao mesmo tempo, permeável aos gases para possibilitar as trocas gasosas necessárias à manutenção da viabilidade.

As espécies frutíferas da biodiversidade requerem um estudo mais aprofundado sobre o menor grau de umidade suportável das sementes, conhecimento sobre qualidade fisiológica e os fatores que contribuem para o comprometimento dessa qualidade, e desta forma adaptar as tecnologias de armazenamento para a espécie. Essas informações viabilizam a conservação durante a secagem parcial, não comprometendo as porcentagens de germinação (BOVI; MARTINS; SPIERING, 2004).

As sementes recalcitrantes com teores elevados de água, são insuficientes para viabilizar a germinação, no entanto tem concedido resultados favoráveis para o desempenho fisiológico das sementes de Euterpe oleracea Mart., que tem subsidiado estudos sobre a conservação de sementes dessa espécie (LIMA, et al., 2018).

A seleção de embalagens considera condições adequadas ao armazenamento que não comprometam a qualidade da semente. E entre os fatores que são levados em consideração nas embalagens são o material a ser utilizado, sendo que este pode ser classificado em porosos que permitem as trocas com o meio, as semipermeáveis que permitem uma troca limitada com o meio e as impermeáveis que não permitem trocas com o meio, essas características possibilitam melhores condições de armazenamento, não comprometendo a disponibilidade comercial e a qualidade da semente (CARVALHO e NAKAGAWA, 2012).

\section{METODOLOGIA}

Os frutos de Bacabi foram coletados no município de Santo Antônio do Tauá/Pará, em uma propriedade rural. O municipio pertence à mesorregião Metropolitana de Belém e à microrregião de Castanhal, onde o clima predominante da área de abrangência é, dada pela classificação de Koppen, do tipo Af, do subtipo úmido - clima tropical chuvoso, as temperaturas médias anuais estão em $25^{\circ} \mathrm{C}$, já a máxima e a mínima, variando de 34 e $24^{\circ} \mathrm{C}$, respectivamente (ALVARES, 2014). 
Os frutos foram colhidos na propriedade a partir de uma matriz com cachos maduros. Posteriormente o material foi transportado para o Laboratório de Tecnologia de Sementes da Universidade Federal Rural da Amazônia em Belém (UFRA), Campus Belém, Pará, Brasil, onde se conduziu o experimento.

Os frutos foram beneficiados imersos por 24 horas em água na temperatura ambiente, para o processo de despolpamento, lavado com água corrente e imerso novamente em água por 30 minutos, em seguida foram selecionadas 3360 sementes para serem colocadas em recipientes como sacos de plástico, de papel, garrafas de polietileno e frascos de vidro. Utilizou-se delineamento experimental em inteiramente casualizado em esquema fatorial de 7 x 3, (sete tratamento e três períodos), classificados como T1 (frasco vidro), T2 (saco plástico), T3 (saco de papel), T4 (garrafa pet), T5 (saco plástico com areia), T6 (saco plástico com serragem ), e T7 (saco plástico preto) em três períodos de armazenamentos (15, 57 e 99 dias). Cada tratamento continha 4 repetições, e 120 sementes em cada repetição, totalizando 480 sementes em cada um dos 7 tratamentos, avaliados nos três períodos.

Antes do armazenamento das sementes em cada recipiente, as mesmas foram submetidas ao teste de umidade para controle e comparação dos períodos posteriores relacionados ao armazenamento, utilizando o método da estufa $\left(105 \pm 3{ }^{\circ} \mathrm{C}\right)$, indicado pelas Regras Brasileiras para Análises de Sementes (BRASIL, 2009) como padrão. Para o teste de germinação as sementes foram então distribuídas em 8 bandejas de plástico com substrato vermiculita umedecida, dispostas aleatoriamente para que houvesse casualização, e semeadas em 4 fileiras com 25 sementes colocadas em posição longitudinal. O semeio foi realizado a partir de quatro repetições de 25 sementes, os tratamentos foram mantidos à temperatura ambiente.

A determinação do teor de água na semente, foi efetuado pelo método estufa a $105 \pm$ $3^{\circ} \mathrm{C}$, durante 24 horas, conforme recomendações das Regras para Análise de Sementes (BRASIL, 1992). Foram utilizadas 1 amostra de 5 sementes para cada repetição dos 7 tratamentos. Os resultados foram expressos em porcentagem média para cada local de armazenamento.

Além de determinado a percentagem de sementes germinadas e não germinadas após os três períodos de análise por contagem manual, consideradas germinadas apenas aquelas que já apresentavam raiz e parte aéreas bem desenvolvidas

A análise estatística das características avaliadas neste experimento foi efetuada utilizando-se o programa estatístico $R$, através de testes de significância e interação entre os fatores para cada variável, as médias dos resultados foram comparadas pelo teste de Tukey 
$(\mathrm{p} \leq 0,05)$. Os Dados foram obtidos a partir das análises realizadas dos três períodos de armazenamento.

\section{RESULTADOS E DISCUSSÃO}

Na Tabela 01 estão apresentados os resultados de interação entre os tratamentos e os períodos de armazenamento em dias, pode-se observar que a média do primeiro período (15 dias), apresenta um valor de umidade acima de $41,77 \%$ para os tratamentos nos recipientes $\mathrm{T} 1$ vidro, T2 saco plástico, T4 garrafa pet, T5 saco plástico com areia, T6 saco plástico com serragem e T7 saco plástico preto. Resultados corroboram aos encontrados por Nascimento et al. (2010), que trabalhou com sementes de E. oleracea, armazenadas em sacos de polietileno sob diferentes temperaturas, os dados do experimento apresentaram uma média de teor de água de cerca de 43,4\% após 90 dias de armazenamento. Já os tratamentos em sacos plásticos com serragem úmida, o autor obteve 43,30\% e em saco de papel 33,30\% de umidade.

Segundo Nascimento e Moraes (2011), realizando estudos com Euterpe oleracea, encontrou uma variação de $10,61 \%$ a $22,30 \%$, de umidade nas sementes ao serem armazenas em diferentes recipientes do tipo fechada em bandejas de isopor, exposto em bandejas plásticas e juta, e para este autor, esses valores variaram, mas que podem apresentar dificuldades no armazenamento e perdas ao agricultor.

De acordo com Crochemore (1993), durante a utilização de embalagens permeáveis há penetração do vapor d'água que acompanham as flutuações de umidade ocorridas no exterior. Tal fato reforça a ideia de que um bom armazenamento isola a semente recalcitrante do meio exterior e impede a troca de água e gases com o meio externo, ocasionando uma boa conservação, prolongando o tempo de vigor da mesma.

Os dados foram estatisticamente iguais, para os períodos de 57 e 99 dias de armazenamento das sementes, com médias 35,58 e 34,16\%, respectivamente, esse comportamento confirma o trabalho de Silva et al. (2010), que argumentou o fato do teor de umidade das sementes recalcitrantes ser maior nos primeiros dias de armazenamento e vai reduzindo com o passar do tempo.

No tratamento T6 (saco plástico com serragem), foi observado somente valores de umidade no primeiro período de armazenamento, pois a partir de 57 dias as sementes já apresentavam $100 \%$ de germinação, deste modo sendo impossível determinar sua umidade. 
BRITO, et al.

Martins et al. (2003), trabalharam com armazenamento de sementes de Palmeira Real Australiana (Archontophoenix alexandrae), pertencentes ao grupo das recalcitrantes e obtiveram teores de água abaixo de $31,5 \%$, observaram que ocorreu uma redução significativa na porcentagem de germinação e até mortalidade total. Ferreira e Santos (1992), observaram que o vigor e a viabilidade de sementes de pupunha (Bactris gasipaes Kunth), após terem sido submetidas à um período de secagem de 15 dias, o teor de umidade foi reduzido de $45 \%$ a $22 \%$ afetando sua emergência e vigor. O comportamento recalcitrante das sementes de $O$. mapora, também foi observado por Probert; Longley (1989), que concluíram que há uma perda da viabilidade quando os níveis de umidade são inferiores a $20 \%$.

Tabela 01. Efeitos da interação entre tratamentos e período de armazenamento em dias no TA\% (Teor de água) das sementes de Oenocarpus mapora coletadas em Santo Antônio do Tauá/Belém/Pará.

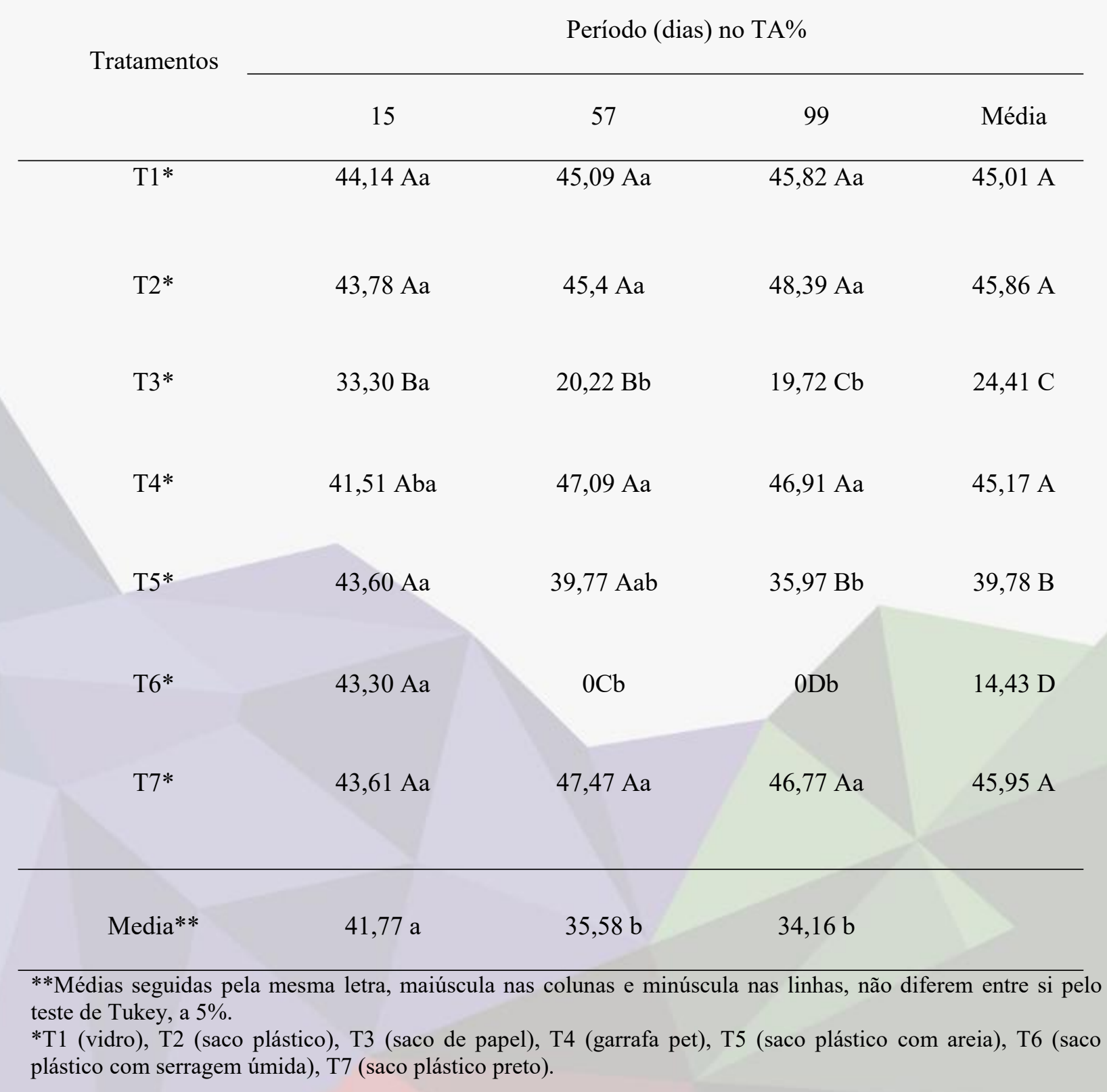


Fonte: Autores (2020)

A Tabela 02, expressa os valores de interação entre os Tratamentos e Períodos (T x P) sobre a percentagem de germinação e não germinação, observamos que os tratamentos T2 (Saco de Plástico), T4 (garrafa PET) e T8 (Saco de plástico preto), apresentam valores de G\% germinação e GN\% não germinação, estatisticamente iguais segundo o teste F. Assim apresentando boa germinação após armazenamento. Vale ressaltar que T6 (Saco de plástico com serragem) foi o tratamento que apresentou o melhor desempenho, com os maiores valores de germinação, no entanto esse fato ocorreu por já estarem germinadas desde o armazenamento.

T5 (Saco com areia) mostrou-se como um bom tipo de armazenamento, no entanto, a partir do terceiro período, se mostrou inviável,

O tratamento T1 apresenta germinação de 100\% apenas no primeiro período, em seguida mostra-se ineficiente, T3 e T7 mostraram-se impróprios pois as sementes vão perdendo sua viabilidade gradativamente.

Tabela 02: Efeitos da interação Local de armazenamento x Tempo de armazenamento em dias $(15,57,99)$ na percentagem de Germinação ( $\mathrm{G} \%$ ) e não Germinação (NG\%) de Sementes de Bacabi armazenadas em oito recipientes: (T1) vidro, (T2) saco plástico, (T3) saco de papel, (T4) garrafa pet, (T5) saco plástico com areia, (T6) saco plástico com serragem úmida, (T7) saco plástico geladeira, (T8) saco plástico preto.

\section{Período (dias)}

Tratamento

15

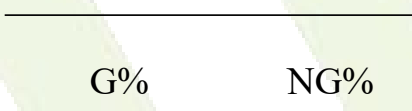

$\mathrm{T} 1$

$\mathrm{T} 2$

T3

T4

T5
$100 \mathrm{Aa}$

$100 \mathrm{Aa}$

$0 \mathrm{Ca}$

$48 \mathrm{Ca}$

$52 \mathrm{Ab}$

$100 \mathrm{Aa}$

$0 \mathrm{Ca}$

100 Aa

$0 \mathrm{Cc}$

$80 \mathrm{Bb}$

$0 \mathrm{Db}$

$0 \mathrm{Db}$
57

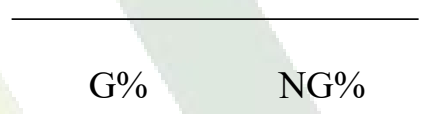

$100 \mathrm{Aa}$

$0 \mathrm{Da}$

$100 \mathrm{Aa}$

$100 \mathrm{Aa}$

$0 \mathrm{Da}$

$20 \mathrm{Cb}$
$100 \mathrm{Aa}$

99

\begin{tabular}{ll}
\hline $\mathrm{G} \%$ & $\mathrm{NG} \%$ \\
\hline $0 \mathrm{Cb}$ & $100 \mathrm{Aa}$
\end{tabular}

97 Aa

$3 \mathrm{Ca}$

\section{$0 \mathrm{Cb}$}

$96 \mathrm{Aa}$

$4 \mathrm{Ca}$ 


\begin{tabular}{lrccccr}
\hline T6 & $100 \mathrm{Aa}$ & $0 \mathrm{Cb}$ & $100 \mathrm{Aa}$ & $0 \mathrm{Db}$ & $100 \mathrm{Ab}$ & $0 \mathrm{Cb}$ \\
$\mathrm{T} 7$ & $83 \mathrm{Ba}$ & $17 \mathrm{Bc}$ & $61 \mathrm{Cb}$ & $39 \mathrm{Bb}$ & $0 \mathrm{Cc}$ & $100 \mathrm{Aa}$ \\
$\mathrm{T} 8$ & $100 \mathrm{Aa}$ & $0 \mathrm{Ca}$ & $100 \mathrm{Aa}$ & $0 \mathrm{Da}$ & $98 \mathrm{Aa}$ & $2 \mathrm{Ca}$
\end{tabular}

Médias seguidas pela mesma letra, maiúscula nas colunas e minúscula nas linhas, não diferem entre si pelo testede Tukey, a $5 \%$.

Fonte: Autores (2020)

\section{CONCLUSÕES}

A viabilidade das sementes de Bacabi é afetada pelo tipo de recipiente e pelo período de armazenamento e necessitam de condições mínimas de umidade durante o armazenamento. No processo de armazenamento, a rusticidade da espécie permite que resistam por algum tempo em condições médias de umidade.

Os melhores recipientes para armazenamento das sementes foram o T2 (saco plástico), T4 (garrafa PET), T5 (saco plástico com areia) e T7 (saco plástico preto), pois preservam as qualidades fisiológicas da semente de bacabi durante o armazenamento por mais tempo, sem alterar a germinação posterior.

\section{REFERÊNCIAS}

ALVARES, Clayton Alcarde et al. Koppen's climate classification map for Brazil. Meteorologische Zeitschrift, v. 22, n. 6, p. 711-728, 2014.

BEZERRA, A. M. E.; FILHO, S. M.; FREITAS, J. B. S.; TEÓFILO, E. M. Avaliação da Qualidade das Sementes de Moringa oleifera Lam. Durante o armazenamento. Ciênc. agrotec., Lavras, v. 28, n. 6, p. 1240-1246, nov./dez., 2014.

BONNER, F.T. Storage of seeds: potential and limitations for germoplasm conservation. Forest Ecology and Management, v.35, n.1, p.35-43, 1990.

BORÉM, E.M. Propriedades Físicas e Térmicas dos Frutos e dos Grãos de Café. Póscolheita do café. UFLA, Lavras-MG. p. 631, 2007.

BOVI, M.L.A.; MARTINS, C.C.; SPIERING, S.H. Desidratação de sementes de quatro lotes de pupunheira: efeitos sobre a germinação e o vigor. Horticultura Brasileira, Brasília, v. 22, n. 1, p. 109-112, jan-mar 2004.

BRASIL. Ministério da Agricultura, Pecuária e Abastecimento. Secretaria de Defesa Agropecuária. 2009. Regras para análise de sementes. MAPA, Brasília, Brasil. 395 p. 2009.

BRONZE, A. B. da S.; MOTA, M. G. da C.; OLIVEIRA, M. do S. P. de; REDIG, M. do S. F.; 
DUARTE, E. B. Análise de crescimento de progênies da bacabi (Oenocarpus mapora Kasten) em sistemas agroflorestais. In: CONGRESSO BRASILEIRO DE SISTEMAS AGROFlORESTAIS, 8., 2011, Belém, PA. Anais... Belém, PA: SBSAF: Embrapa Amazônia Oriental: UFRA : CEPLAC : EMATER : ICRAF, 2011.

CARVALHO, J.E.U. MULLER, C.H. NASCIMENTO, W.M.O. Classificação de Sementes de Espécies Frutíferas Nativas da Amazônia de acordo com o comportamento no armazenamento. Comun. téc. n. 60, Belém, 2001.

CAVAlCANTE, P. B. Frutas comestíveis da Amazônia. CEJUP: Museu Paraense Emílio Goeldi, 279 p. Belém, PA: 1991.

CARVALHO, N.M.; NAKAGAWA, J. Sementes. Ciência, Tecnologia e Produção. Funep, Jaboticabal - SP. p. 590, 2012.

CUNHA, A. C. da C.; JARDIM, M. A. G. Avaliação do potencial germinativo em açaí (Euterpe oleracea Mart.). Variedades preto, branco e espada. In: I Reunião dos Botânicos da Amazônia, 26 a 30 jun. Belém: PA, 1995. Boletim do Museu Paraense Emílio Goeldi, sér. Bot. 11(1), p. 55-59, 1995.

CROCHEMORE, M. L. Conservação de sementes de tremoço azul em diferentes embalagens. Revista Brasileira de Sementes, Londrina, v.15, n.2, p.227-232, 1993.

DOMINGUES, Alessandra Ferraiolo Nogueira. Caracterização físico-química da polpa de bacabi (Oenocarpus mapora H. Karsten). Belém, PA: Embrapa Amazônia Oriental, 2014. $18 \mathrm{p}$.

FOWLER, J.A.P. Superação de dormência e armazenamento de sementes de espécies florestais. In: GALVÃO, A.P.M. (Org.) Reflorestamento de propriedades rurais para fins produtivos e ambientais: um guia para ações municipais e regionais. Brasília: Embrapa Comunicação para Transferência de Tecnologia; Colombo, PR: Embrapa Florestas, 2000. p. 77-99.

FERREIRA, S. A. do N. SANTOS, L. A. Viabilidade de Sementes de Pupunha (Bactris gasipaes Kunth). Acta Amazônica, vol. 22, 1992.

GRAHAM, I.A. Seed storage oil mobilization. Annual Review of Plant Biology, v.59, p.115-142, 2008.

HENDERSON, A.; GALEANO, G.; BERNAL, R. Field guide to the Palms of the Americas. New Jersey: Princeton University Press, 1995. 418 p.

MARTINS, C.C.; BOVI, M.L.A.; NAKAGAWA, J. Desiccation effects on germination and vigor of King palm seeds. Horticultura Brasileira, v. 21, n. 1, p. 88-92. Brasília, 2003.

JOSÉ, A. C.; ERASMO, E. A. L.; COUTINHO, A. B. Germinação e tolerância à dessecação de sementes de bacaba (Oenocarpus bacaba Mart.). Revista Brasileira de Sementes, n. 34, p. 651-657, 2012.

LIMA, J.M.E.; OLIVEIRA, J.A.; SMIDERLE, O.J.; LOUSADO, A.V.C.; CARVALHO, M.L.M. Physiological performance of açai seeds (Euterpe oleracea Mart.) stored with diferente moisture contents and treated with fungicide. Journal of Seed Science, v.40, n.2, 
p.135-145, 2018.

MARCOS FILHO, J. Fisiologia de sementes de plantas cultivadas. Piracicaba: FEALQ, 495p, 2005.

MEDEIROS, Antônio Carlos de S. Armazenamento de sementes de espécies florestais nativas: Embrapa Florestas, 2001. 24 p.

MENDONÇA, A. P. CORREIA, G.A. MORAES, L.I. ARAÚJO, M.E. LIMA, Y.T.T.B. Armazenamento de Açaí (Euterpe oleracea Mart.). 64 Reunião Anual da SBPC, São Luís, MA, 2012.

MIRANDA, I. P. A.; RABELO, A.; BUENO, C. R.; BARBOSA, E. M.; RIBEIRO, M. N. S.; frutos de palmeiras da Amazônia. Manaus, 2001.

MOURA, E.F. OLIVEIRA, S.P. SILVA, D.T. PONTES, L.C.G. Genetic Diversity and Structure of Oenocarpus mapora Germplasm Conserved at Eastern Amazon. Rev. Bras. Frutic., Jaboticabal - SP, v. 37, n. 4, p. 984-992, Dezembro, 2015.

NAKAGAWA, J. Testes de vigor baseados na avaliação das plântulas. In: VIEIRA, R.D.; CARVALHO, N.M. Teste de vigor em sementes. Jaboticabal, FUNEP, 1994. p.49-85.

NASCIMENTO, W. M. O.; MORAES, M. H. D. Fungos associados a sementes de açaí: efeito da temperatura e do teor de água das sementes durante o armazenamento. Revista Brasileira de Sementes, v. 33, n.3, p. 415-425, 2011.

NASCIMENTO, W. M. O. do; CICERO, S. M.; NOVEMBRE, A. D. da L. C. Conservação de sementes de açaí (Euterpe oleracea Mart.). Revista Brasileira de Sementes, Pelotas, v. 32, n. 1, p. 24-33, 2010.

NASCIMENTO, W. M. O. Conservação de sementes de açaí (Euterpe oleracea Mart.). 2006. 60 f. Tese (Doutorado em Agronomia/Fitotecnia) - Escola Superior de Agricultura Luiz de Queiroz, Universidade de São Paulo, Piracicaba, 2006.

OLIVEIRA, M.S.P. RIOS, S.A. Potencial Econômico de Algumas Palmeiras da Amazônia. In: VI Encontro Amazônico de Agrárias, Belém- PA, 2014. Anais Encontro Amazônico de Agrárias, Belém- PA, 2014.

OLIVEIRA, M. S. P.; MOURA, E. F. Repetibilidade e número mínimo de medições para caracteres de cacho de bacabi (Oenocarpus mapora). Revista Brasileira de Fruticultura, v. 32, n. 4, p. 1173-1179, dez. 2010.

OLIVEIRA, M. S. P. DO; PADILHA, N. C. C.; FERNANDES, T. S. D. Ecologia da polinização de Oenocarpus mapora Karsten (ARECACEAE) nas condições de Belém (PA). Revista Ciências Agrária, n 38, p 91-106, Belém, 2002.

PROBERT, R.J.; LONGLEY, P.L. Recalcitrant seed storage physiology in three aquatic grasses (Zizania palustris, Spartina anglica and Portesia coarctata). Annals of Botany, v. 63, n. 1, p. 53-63, 1989.

QUEIROZ, M. S. M.; BIANCO, R. Morfologia e desenvolvimento germinativo de Oenocarpus bacaba Mart. (Arecaceae) da Amazônia Ocidental. Revista Árvore, v. 33, n. 6, 
p. 1037-1042, nov./dez. 2009.

ROBERTS, E. H. (ed.) Viabilty Of Seends. Chapman and Hall Limited, London, 1972.

RODRIGUES, A. E. CARVALHO, J.E.U. MULLER, C.H. NASCIMENTO, W.M.O. Classificação de Sementes de Espécies Frutíferas Nativas da Amazônia de acordo com o comportamento no armazenamento. Comun. téc. N. 60, Julho/2001, p.1-4.

SILVA, R. J. F. Anatomia foliar comparada em espécies de Oenocarpus Mart. (Arecaceae) de Belém, Pará, Brasil: uma contribuição taxonômica. 2006. 91 f. Dissertação (Mestrado em Botânica) - Universidade Federal Rural da Amazônia, Belém, PA.

SILVA, Ricardo Alexandre Moraes da; MOTA, Milton Guilherme da Costa; FARIAS NETO, João Tomé de. Emergência e crescimento de plântulas de bacabi (Oenocarpus mapora Karsten) e bacaba (Oenocarpus distichus Mart.) e estimativas de parâmetros genéticos. Acta Amaz., Manaus, v. 39, n. 3, p. 601-608, Sept. 2009.

SILVA, F.S. PORTO, A.G. PASCUALI, L.C. SILVA, F.T.C. Viabilidade do armazenamento de sementes em diferentes embalagens para pequenas propriedades rurais. Revista de Ciências Agro-Ambientais, Alta Floresta, v.8, n.1, p.45- 56, 2010. 